\title{
Disclosing the true nature of the Sy 2 galaxy NGC 3281: One more Compton-thick source
}

\author{
C. Vignali ${ }^{1, \star}$ and A. Comastri ${ }^{1}$ \\ Osservatorio Astronomico di Bologna, via Ranzani 1, 40127 Bologna, Italy
}

Received 31 August 2001 / Accepted 29 October 2001

\begin{abstract}
We present the BeppoSAX broad-band X-ray spectrum of the Seyfert 2 galaxy NGC 3281. The source high-energy spectrum is characterized by the nuclear transmitted component, with an absorbing column density of $\approx 2 \times 10^{24} \mathrm{~cm}^{-2}$, while the MECS spectrum is reflection-dominated, with a prominent $(E W \approx 0.5-1.2 \mathrm{keV})$ iron $\mathrm{K} \alpha$ emission line. The source is detected at only the $5 \sigma$ significance level in the LECS band, because of the strong obscuration which hampers at low energies the direct view of the active nucleus harbored in NGC 3281. BeppoSAX results are consistent with the scenario where NGC 3281 is inclined more than $60^{\circ}$ with respect to the line-of-sight. Combining the $N_{\mathrm{H}}$ value obtained from the present $\mathrm{X}$-ray analysis with the $A_{\mathrm{V}}$ measurement, a $N_{\mathrm{H}} / A_{\mathrm{V}}$ about 50 times the Galactic value is derived.
\end{abstract}

Key words. galaxies: Seyferts: individual: NGC 3281 - galaxies: nuclei - galaxies: active - X-rays: galaxies

\section{Introduction}

According to the unified models (e.g., Antonucci 1993), Seyfert 2 galaxies are powered by the same engine of Seyfert 1s but are seen at medium/high inclination angles with respect to the line-of-sight, thus being absorbed by thick matter in the equatorial plane. This picture, at least at its first order approximation, has found strong support from many observational evidences, including optical spectropolarimetry (e.g., Antonucci \& Miller 1985; Miller \& Goodrich 1990), near-infrared spectroscopy (e.g., Goodrich et al. 1994) and X-ray spectroscopy (e.g., Turner et al. 1997a,b, 1998). For column densities lower than $10^{24} \mathrm{~cm}^{-2}$, the $\mathrm{X}$-rays above a few $\mathrm{keV}$ are able to penetrate the absorbing medium (likely to be associated to the torus) and to be detectable as direct continuum emission (Compton-thin sources). If the absorbing column density exceeds $\sim 1.5 \times 10^{24} \mathrm{~cm}^{-2}$, then the nuclear radiation is obscured up to about $10 \mathrm{keV}$ and the source is called Compton-thick (see Matt et al. 2000 and Matt 2001 for the most recent reviews). While in a handful of Compton-thick sources the column density has been directly measured (e.g., NGC 6240: Vignati et al. 1999; NGC 4945: Iwasawa et al. 1993; Done et al. 1996;

Send offprint requests to: C. Vignali, e-mail: chris@astro.psu.edu

* Present address: Department of Astronomy and Astrophysics, The Pennsylvania State University, 525 Davey Lab, University Park, PA 16802, USA.
Guainazzi et al. 2000; Madejski et al. 2000; Circinus: Matt et al. 1999a), in the majority of them the column density is so large to totally obscure the nucleus even in the hard X-ray band (e.g., NGC 1068: Matt et al. 1997; NGC 7674: Malaguti et al. 1998) or their flux at high energies is too low to allow a detailed spectral analysis (Salvati et al. 1997; Maiolino et al. 1998).

Emission from Compton-thick galaxies can be detected by means of the reflection caused by the visible inner surface of the torus and/or via scattering by the material responsible for the broad lines observed in polarized light (e.g., Antonucci \& Miller 1985; Tran 1995). In the X-ray band, the spectrum of Compton-thick sources is generally characterized by an iron $\mathrm{K} \alpha$ line with extremely large equivalent width $(E W \gtrsim 1 \mathrm{keV})$ and a reflection-dominated continuum flatter than the intrinsic spectrum (e.g., Maiolino et al. 1998). In the last few years, thanks to the high capabilities of observing Comptonthick sources with broad-band instruments like those onboard ASCA (e.g., Matt et al. 1996a; Collinge \& Brandt 2000), RXTE (e.g., Madejski et al. 2000), BeppoSAX (e.g., Malaguti et al. 1998; Matt et al. 1999a; Cappi et al. 1999; Vignati et al. 1999; Guainazzi et al. 2000; Franceschini et al. 2000; Iwasawa et al. 2001) and, more recently, $X M M-N e w t o n$ (Guainazzi et al., in preparation), much has be learned about their X-ray properties. However, there are still several astrophysical reasons why Compton-thick sources deserve to be studied in detail. About $50 \%$ of the active galactic nuclei (AGNs) in the local Universe are 
obscured by Compton-thick matter (e.g., Maiolino et al. 1998; Risaliti et al. 1999). Even though their contribution to the $\mathrm{X}$-ray background (XRB) is not energetically dominant (unless the number of such objects is highly underestimated), they may constitute however an important ingredient for the IR and the sub-mm backgrounds, where most of the absorbed radiation is re-emitted by dust (Fabian \& Iwasawa 1999; Brusa et al. 2001). Furthermore, Compton-thick sources allow to study those spectral components that otherwise would be dominated by the nuclear continuum emission (Matt et al. 1996b; Bianchi et al. 2001).

In this regard, NGC 3281 is an extremely interesting object. $A S C A$ observation, in a relatively short exposure time $(\approx 15 \mathrm{ks})$, revealed a complex $\mathrm{X}$-ray spectrum with a prominent iron line (equivalent width of $\left.\sim 480_{-230}^{+770} \mathrm{eV}\right)$ and a large absorbing column density $\left(N_{\mathrm{H}} \simeq 7.1 \pm 1.2 \times 10^{23} \mathrm{~cm}^{-2}\right.$, Simpson 1998). The extinction to the nucleus inferred from the near-infrared studies, $A_{\mathrm{V}}=22 \pm 11 \mathrm{mag}$, combined with the $N_{\mathrm{H}}$ measurement derived from the $\mathrm{X}$-ray analysis, gives a $N_{\mathrm{H}} / A_{\mathrm{V}}$ ratio which is more than one order of magnitude larger than the Galactic value $\left(N_{\mathrm{H}} / A_{\mathrm{V}}=1.9 \times 10^{21} \mathrm{~cm}^{-2} \mathrm{mag}^{-1}\right.$, assuming $A_{\mathrm{V}}=3.1 E(B-V)$, Bohlin et al. 1978). Simpson (1998) has explained this finding as due to a dense cloud in the foreground of both the $\mathrm{X}$-ray and the infrared emitting regions that obscures the whole $\mathrm{X}$-ray source but only a fraction of the much larger IR region. There is however increasing evidence from local AGNs (Risaliti et al. 2000; Maiolino et al. 2001a; Severgnini et al. 2001) and optical/X-ray selected quasars (Risaliti et al. 2001; Fiore et al. 2000; Comastri et al. 2001) that the $N_{\mathrm{H}} / A_{\mathrm{V}}$ ratio can be much higher than the Galactic value. This ratio may also affect the fraction of nuclear radiation reprocessed and re-emitted by dust at far-infrared and sub-millimeter wavelengths.

\section{Observation and data reduction}

The Italian-Dutch satellite BeppoSAX (Boella et al. 1997a) carries four co-aligned Narrow-Field Instruments (hereafter NFI), two of which are gas scintillation proportional counters with imaging capabilities: the Low Energy Concentrator Spectrometer (LECS: 0.1-10 keV, Parmar et al. 1997) and the Medium Energy Concentrator Spectrometer (MECS: 1.3-10 keV, Boella et al. 1997b). The remaining two instruments are the High Pressure Gas Scintillation Proportional Counter (HPGSPC: 4-120 keV, Manzo et al. 1997), which was switched off during the observation, and the Phoswich Detector System (PDS: 13-200 keV, Frontera et al. 1997).

NGC 3281 was observed by BeppoSAX from May 20 to May 23 2000. Standard reduction techniques and screening criteria were applied in order to produce useful linearized and equalized event files. The resulting net exposure times are about $71.3 \mathrm{ks}, 13.5 \mathrm{ks}$ and $35 \mathrm{ks}$ for MECS, LECS and PDS, respectively. Spectra from MECS and LECS were extracted from circular regions of radius $4^{\prime}$ around the source centroid, while background spectra were extracted from both blank-sky event files and from source-free regions in the target field-of-view, with no apparent difference between the two spectra. The background subtraction in PDS light curves and spectra was performed by plain subtraction of the "offsource" from the "on-source" products. The systematic uncertainties of this method are lower than $\simeq 0.03 \mathrm{~s}^{-1}$ in the full PDS sensitive energy bandpass (Guainazzi \& Matteuzzi 1997). The background-subtracted count rates are $2.19 \pm 0.06 \times 10^{-2}, \quad 3.36 \pm 0.63 \times 10^{-3}$ and $0.73 \pm 0.03$ counts per second for MECS $(1.3-10 \mathrm{keV}$ band), LECS (0.5-4.5 keV) and PDS (13-100 keV), respectively.

The choice of the extracting regions is such to ensure that the groups of galaxies populating NGC 3281 field-ofview (Ferguson \& Sandage 1990) and apparent in MECS image have a very poor contribution (if any) to the source spectrum in the $E<10 \mathrm{keV}$ energy range. The PDS band, owing to the soft, presumably thermal spectra characterizing these groups of galaxies, is totally unaffected. A flatspectrum radio-loud quasar is present in PDS field-of-view (PKS 1030-357). The extrapolation of its radio flux to the X-ray band by means of a power law well matches the ROSAT All Sky Survey (RASS) detection and gives a very poor contribution, of the order of a few $10^{-3}$, to the 20-100 keV flux. No known bright source is present in the PDS field-of-view. The probability of a serendipitous source with a flux equal or larger than NGC 3281 is $\sim 3.9 \times 10^{-3}$ (assuming the $A S C A 2-10 \mathrm{keV} \log N-\log S$ from Cagnoni et al. 1998).

No significant flux variability has been revealed either in the MECS or in the PDS light curve.

The spectral analysis was carried out through the XSPEC program (version 11, Arnaud 1996). Errors are quoted at $90 \%$ confidence level for one interesting parameter $\left(\Delta \chi^{2}=2.71\right.$, Avni 1976), and energies are reported in the source rest frame. The Anders \& Grevesse (1989) abundances have been used throughout the paper, as well as a cosmology with $H_{0}=70 \mathrm{~km} \mathrm{~s}^{-1} \mathrm{Mpc}^{-1}$ and $q_{0}=0.5$ (unless otherwise stated).

\section{Spectral analysis}

NGC 3281 is detected in the LECS at only the $\approx 5 \sigma$ significance level, the cause being the short exposure time in the low-energy spectrometer (about $13.5 \mathrm{ks}$ ) and presumably the large absorption obscuring the source, which previous ROSAT and Einstein IPC (Fabbiano et al. 1992) observations have already suggested. Due to the low quality of the data in the LECS band, in the following only the MECS and PDS data will be presented. After an introduction to the broad-band X-ray spectrum of NGC 3281 (Sect. 3.1) using the 1.3-100 keV MECS+PDS data, we will focus on the $3-100 \mathrm{keV} \mathrm{X}$-ray properties (Sect. 3.2). Then the results will be discussed and put into a physically more exhaustive scenario (Sect. 4). 


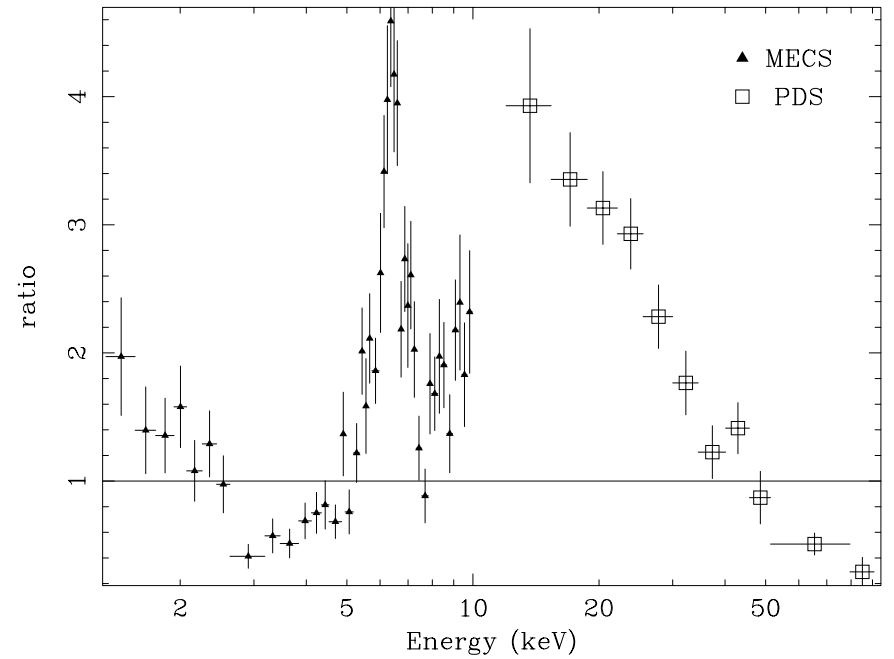

Fig. 1. Residuals of the MECS+PDS 1.3-100 keV spectrum for a model consisting of a single power law with $\Gamma \sim 0.3$ and Galactic absorption. Spectral complexity in the soft X-ray band is clearly present, as well as a strong Fe $\mathrm{K} \alpha$ emission line at about $6.4 \mathrm{keV}$ and an upturn of the spectrum in the PDS band.

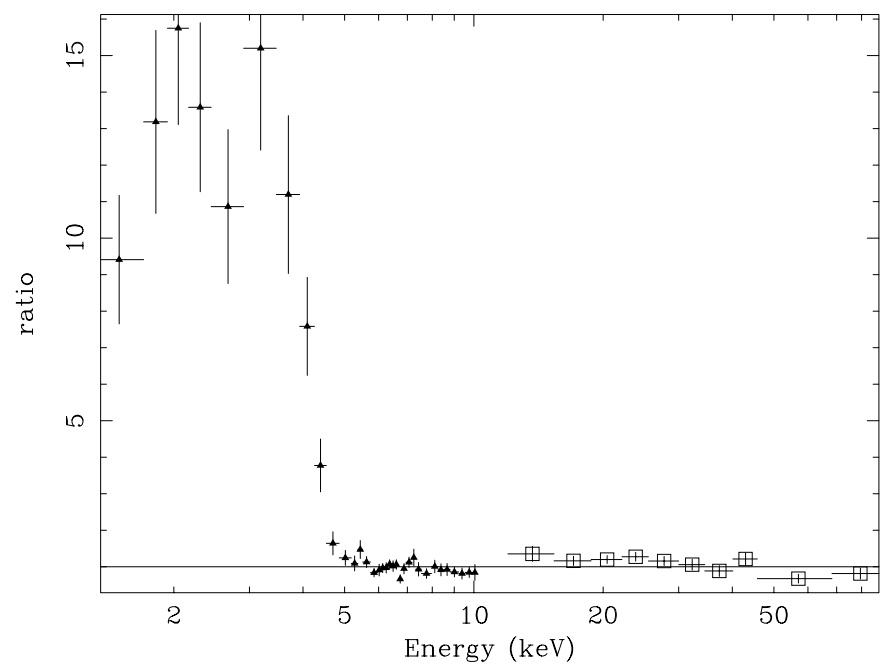

Fig. 2. The residuals obtained assuming a transmission plus iron $\mathrm{K} \alpha$ emission line model.

\subsection{An introduction to the source spectral complexity: The 1.3-100 keV spectrum}

To allow for differences in the absolute flux calibration of the MECS and PDS detectors, the normalizations of the two instruments were allowed to vary within the fiducial values reported by Fiore et al. (1999). A column density of $6.42 \times 10^{20} \mathrm{~cm}^{-2}$, due to absorption by the Galaxy (Dickey \& Lockman 1990), is included in all of the models. In Fig. 1 the residuals obtained by fitting the $1.3-100 \mathrm{keV}$ spectrum with a power-law model plus Galactic absorption are shown. The fit $\left(\chi^{2}=764\right.$ with 96 degrees of freedom) provides an extremely flat slope $(\Gamma \simeq 0.3)$ and clearly illustrates the complex spectral structure below $10 \mathrm{keV}$ and the sharp "rise and fall" of the spectrum at energies above $\approx 8 \mathrm{keV}$. The shape of the residuals suggests the presence

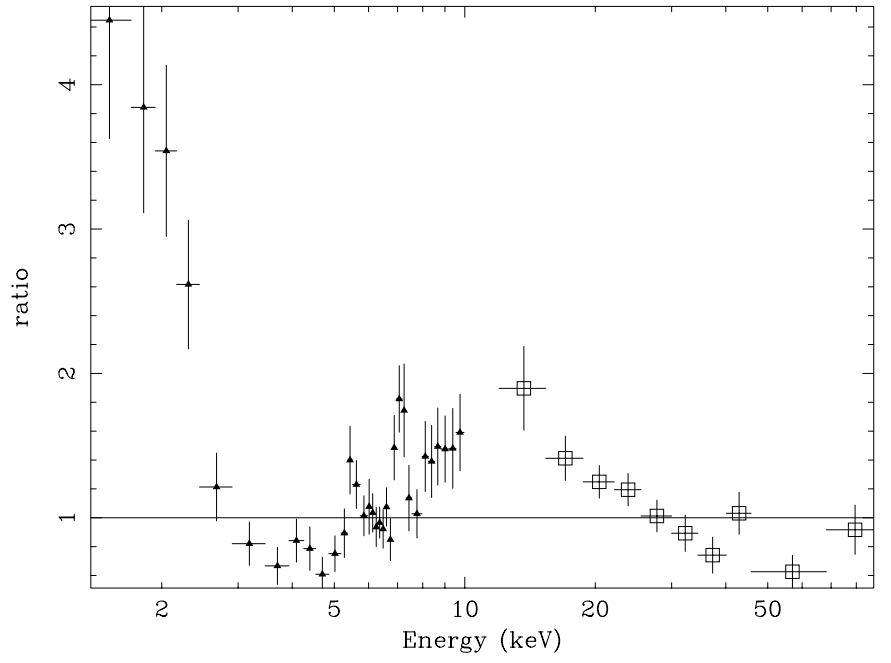

Fig. 3. The residuals obtained assuming a reflection plus iron $\mathrm{K} \alpha$ emission line model.

of a nuclear, absorbed component, which is likely to dominate the emission in the PDS band. We therefore tried to fit the data with an absorbed power-law model (transmission model) plus a narrow iron $\mathrm{K} \alpha$ emission line. The large excess of counts below $\approx 5 \mathrm{keV}$ (Fig. 2 ) strongly indicates that this is only a rough parameterization of the spectrum of NGC $3281\left(\chi^{2} /\right.$ d.o.f. $\left.=311 / 93\right)$, and that the emission in the MECS band has probably a different origin.

Therefore, owing to the flat spectrum and the presence of a strong $\mathrm{Fe} \mathrm{K} \alpha$ emission line, we tried to fit the overall broad-band spectrum with a pure-reflection model (PEXRAV in XSPEC, Magdziarz \& Zdziarski 1995). Although this model provides a statistically significant improvement in the spectral fit with respect to the transmission model $\left(\Delta \chi^{2} \simeq 74\right.$ with the same number of d.o.f., corresponding to an improvement $>99.99 \%$ according to the F-test), it still represents a bad parameterization of NGC $3281 \mathrm{X}$-ray emission. Indeed, large residuals appear evident (Fig. 3), both in the MECS and in the PDS band. An additional absorbed power-law component is likely to be responsible for most of the $\mathrm{X}$-ray emission in the PDS band (see Sect. 3.2).

However, neither the transmission nor the reflection model (or a combination of the two) are able to reproduce the soft $(E \lesssim 3 \mathrm{keV}) \mathrm{X}$-ray spectrum of NGC 3281 . The deficit of counts in the $2-4 \mathrm{keV}$ energy range, coupled with the excess of counts below $2 \mathrm{keV}$ (Fig. 1), indicates that a more complex model is required. Thermal emission and/or absorption plus emission from a hot, photoionized medium could be relevant for NGC 3281, as well as for Mrk 3, a Compton-thick galaxy which shows a similar spectral behavior (Iwasawa 1995; Cappi et al. 1999). A soft $\mathrm{X}$-ray emission was indeed revealed by $A S C A$ (Simpson 1998 ) thanks to the low-energy spectral coverage down to $\sim 0.5 \mathrm{keV}$ provided by the SIS instrument. Unfortunately, the nature of this component cannot actually be addressed by the present LECS data due to the source low counting statistics in this instrument. It should be noted that soft 
$\mathrm{X}$-ray components are not uncommon in obscured AGNs, as recently observed in Compton-thick sources (Guainazzi et al. 1999) or derived from the $\mathrm{X}$-ray analysis of a large sample of hard X-ray selected AGNs (Vignali et al. 2001 and references therein).

In the following we will discuss only the $3-100 \mathrm{keV}$ spectrum of NGC 3281, demanding the study of the $E<3 \mathrm{keV}$ spectral complexity to other better-suited $\mathrm{X}$-ray instruments, as those onboard Chandra and XMM-Newton.

\subsection{The 3-100 keV spectrum}

Supported by the broad-band spectral analysis presented in Sect. 3.1, a power-law component was added to the transmission plus iron line model to mitigate the residuals in the MECS band below $\approx 5 \mathrm{keV}$ (model (a) in Table 1). While the nuclear transmitted component is characterized by a slope $\left(\Gamma_{\mathrm{H}}=2.07_{-0.27}^{+0.32}\right)$ which is consistent, within the errors, with that typical of AGNs (e.g., Nandra \& Pounds 1994; Piro et al. 2000), the extremely flat power law below $10 \mathrm{keV}\left(\Gamma_{\mathrm{S}}=0.78_{-0.27}^{+0.59}\right)$ and the prominent iron line prompted us to fit the overall broad-band spectrum with the following transmission plus reflection model ((b) in Table 1)

$$
\begin{aligned}
F(E)= & {\left[A_{\mathrm{tr}} \operatorname{Tr}\left(E, N_{\mathrm{H}}, \Gamma_{\mathrm{H}}\right)+A_{\mathrm{refl}} R\left(E, \Gamma_{\mathrm{H}}, E_{\mathrm{c}}\right)\right.} \\
& +\mathrm{K} \alpha \text { emission line }] \mathrm{e}^{-N_{\mathrm{H}, \mathrm{gal}} \sigma_{\mathrm{ph}}}
\end{aligned}
$$

where $\operatorname{Tr}\left(E, N_{\mathrm{H}}, \Gamma_{\mathrm{H}}\right)$ is the power-law component (with slope $\Gamma_{\mathrm{H}}$ ) transmitted through the column density $N_{\mathrm{H}}$, $R\left(E, \Gamma_{\mathrm{H}}, E_{\mathrm{c}}\right)$ is the pure-reflection spectrum obtained using PEXRAV in XSPEC with e-folding energy $E_{\mathrm{c}}, A_{\mathrm{tr}}$ and $A_{\text {refl }}$ are the normalizations of the transmitted and reflected component, respectively, and $N_{\mathrm{H} \text {,gal }}$ is the absorption due to the Galaxy. The reflection is required at a highsignificant level (>99.95\%) according to the F-test. The best-fit photon index, $\Gamma=1.95 \pm 0.18$, is consistent with the values reported for Seyfert 1 galaxies, thus confirming one of the basic expectations of the Unified Models, i.e. the same engine powering both Sey 1 and Sey 2 galaxies. The column density, $N_{\mathrm{H}} \simeq 1.51 \pm 0.20 \times 10^{24} \mathrm{~cm}^{-2}$ (Fig. 4), indicates that NGC 3281 is likely to be a "border-line object" between the Compton-thin and the Compton-thick sources. The angle subtended by the reflecting matter to the nucleus, given by the relative normalization of the reflected versus the direct component (both reported in Table 1 ), is $\approx 0.33 \times 2 \pi$. The iron $\mathrm{K} \alpha$ line rest-frame energy, $6.37 \pm 0.07 \mathrm{keV}$, is consistent with a neutral or mildly ionized origin, and its equivalent width $(E W \approx 1.2 \mathrm{keV})$, although not so extreme for a candidate Compton-thick galaxy, is consistent with the amount of reflection derived from the spectral fit. This model provides a good fit to the broad-band data (Fig. 5) and the residuals do not indicate evident additional spectral components. Small residuals at $\approx 7 \mathrm{keV}$ were fitted with an additional emission line, which could be associated to either the ionized H-like iron line arising from ionized matter (the "warm mirror" observed in several Compton-thick galaxies; e.g.,

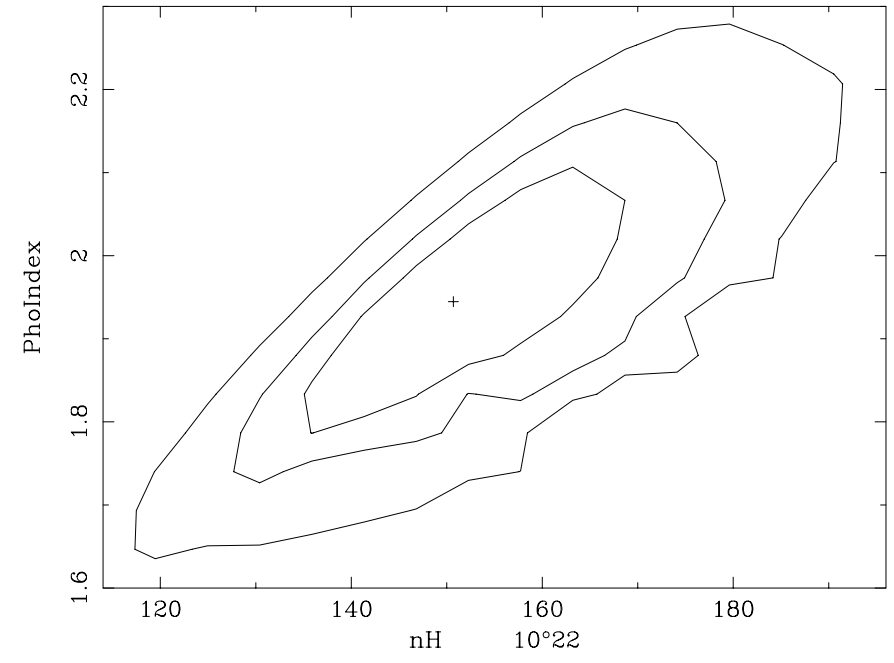

Fig. 4. BeppoSAX 68, 90 and $99 \%$ confidence contours relative to the power-law slope $\Gamma$ vs. the column density $N_{\mathrm{H}}$ (model (b) in Table 1).

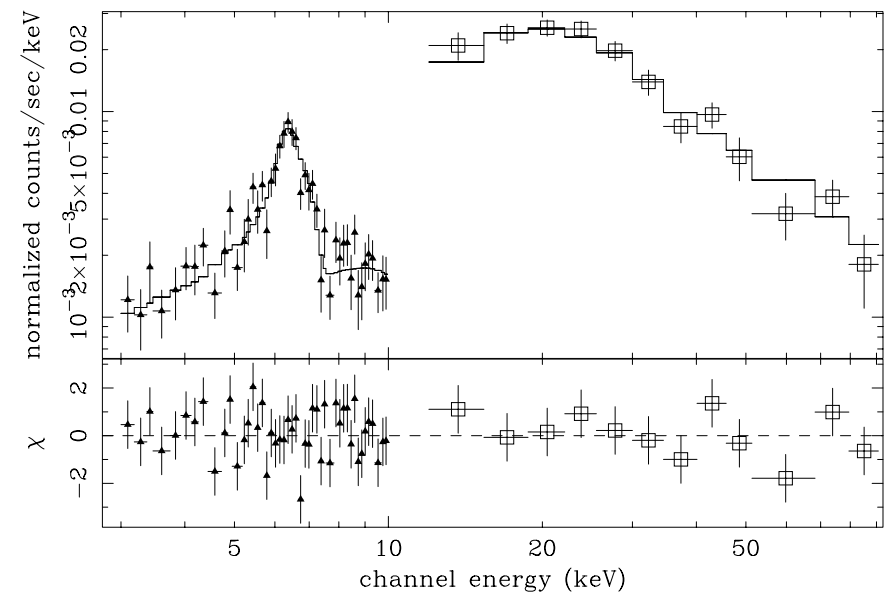

Fig. 5. BeppoSAX MECS+PDS 3-100 keV spectrum of NGC 3281 (model (b) in Table 1).

Matt et al. 1999, 2000) or to the $7.06 \mathrm{keV}$ iron $\mathrm{K} \beta$ emission line revealed in a few Compton-thick sources (e.g., Matt et al. 1996a; Malaguti et al. 1998). However, this feature is not statistically significant, its EW upper limit being of $610 \mathrm{eV}$.

We also tried a different scenario, in which the reflecting matter is partially covered. This would represent a situation in which the line-of-sight towards the nucleus is heavily blocked due to absorption, but the optical depth diminishes at higher torus latitudes, thus allowing transmission of some of the radiation reflected by the far side of the torus. With the present BeppoSAX data, no apparent difference between an unabsorbed reflection and an absorbed one was found in either the $\mathrm{X}$-ray spectral fits or in the derived parameters.

Column densities of the order of $10^{24} \mathrm{~cm}^{-2}$ imply that the photons which eventually emerge may have been suffered one or more Compton scatterings. Therefore we adopted the model of Matt et al. (1999b), based on MonteCarlo simulations, which properly treats the photon 
Table 1. MECS+PDS: 3-100 keV spectral fits.

\begin{tabular}{lcccccccc}
\hline Model & $\Gamma_{\mathrm{S}}$ & $\Gamma_{\mathrm{H}}$ & $\begin{array}{c}N_{\mathrm{H}} \\
\left(\times 10^{24} \mathrm{~cm}^{-2}\right)\end{array}$ & $\begin{array}{c}A_{\mathrm{tr}} \\
\left(\times 10^{-2}\right)\end{array}$ & $\begin{array}{c}A_{\text {refl }} \\
\left(\times 10^{-3}\right)\end{array}$ & $\begin{array}{c}E_{\mathrm{K} \alpha} \\
(\mathrm{keV})\end{array}$ & $\begin{array}{c}E W_{\mathrm{K} \alpha} \\
(\mathrm{eV})\end{array}$ & $\chi^{2} /$ d.o.f. \\
\hline (a) & $0.78_{-0.27}^{+0.59}$ & $2.07_{-0.27}^{+0.32}$ & $1.51_{-0.24}^{+0.26}$ & $3.42_{-2.00}^{+5.91}$ & & $6.36_{-0.07}^{+0.06}$ & $2340_{-710}^{+750}$ & $78.9 / 65$ \\
(b) & & $1.95 \pm 0.18$ & $1.51_{-0.19}^{+0.20}$ & $2.24_{-1.13}^{+2.03}$ & $7.41_{-2.04}^{+2.60}$ & $6.37 \pm 0.07$ & $1180_{-361}^{+400}$ & $69.7 / 66$ \\
(c) & & $1.98_{-0.21}^{+0.04}$ & $1.96_{-0.05}^{+0.19}$ & $5.26_{-0.40}^{+3.29}$ & $6.84_{-2.04}^{+0.96}$ & $6.52 \pm 0.06$ & $526_{-144}^{+128}$ & $83.3 / 66$ \\
\hline
\end{tabular}

transfer in very high-column density matter, including the Compton down-scattering and the Klein-Nishina decline, and assuming a spherical geometry for the distribution of the matter (model (c) in Table 1). By this model the column density increases $\left(N_{\mathrm{H}}=1.96_{-0.05}^{+0.19} \times 10^{24} \mathrm{~cm}^{-2}\right)$, while both the solid angle subtended by the reflecting matter to the nucleus $(\approx 0.13 \times 2 \pi)$ and the iron $\mathrm{K} \alpha$ line equivalent width $(\approx 500 \mathrm{eV})$ decrease. This is due to the increased strength of the nuclear transmitted component with respect to the reflected one, as shown in Table 1. Even though the fit obtained with such a model is statistically worse $\left(\chi^{2}=83.3 / 66\right)$, it must be considered physically more correct than those described above, due to the particular treatment of the photon transfer required in this heavily absorbed environment.

Only a lower limit $(\approx 60 \mathrm{keV})$ was derived for the highenergy cut-off. The observed $2-10 \mathrm{keV}$ and $20-100 \mathrm{keV}$ fluxes of NGC 3281 derived from the best-fit model are $\approx 2.9 \times 10^{-12}$ and $7.2 \times 10^{-11} \mathrm{erg} \mathrm{cm}^{-2} \mathrm{~s}^{-1}$, respectively. These fluxes correspond to $2-10 \mathrm{keV}$ and $20-100 \mathrm{keV}$ intrinsic luminosities of $\approx 1.6 \times 10^{43}$ and $2.0 \times 10^{43} \mathrm{erg} \mathrm{s}^{-1}$, respectively $\left(\approx 3.1 \times 10^{43}\right.$ and $3.9 \times 10^{43} \mathrm{erg} \mathrm{s}^{-1}$ adopting $H_{0}=50 \mathrm{~km} \mathrm{~s}^{-1} \mathrm{Mpc}^{-1}$ and $\left.q_{0}=0\right)$. The $2-10 \mathrm{keV}$ flux obtained with $A S C A$ data in January 1996 observation (Simpson 1998) is fully consistent with that derived by BeppoSAX.

\section{Discussion}

Thanks to its broad-band capabilities, BeppoSAX has been able to reveal the $\mathrm{X}$-ray spectrum of the Sey 2 galaxy NGC 3281 being more complex than previously derived from $A S C A$ observation (due to its limited bandpass). Below $10 \mathrm{keV}$, the typical imprints of the reflected continuum (i.e., a flat slope and a moderately strong iron $\mathrm{K} \alpha$ emission line) do clearly appear, and the presence of a heavily absorbed component is suggested. The reflecting matter appears to be neutral or only marginally ionized, since there is no clear evidence of ionized iron features.

In the PDS band the nuclear, heavily absorbed continuum $\left(N_{\mathrm{H}} \simeq 2 \times 10^{24} \mathrm{~cm}^{-2}\right)$ is able to emerge and to reveal the true nature of NGC 3281, being a Comptonthick source. Due to the broad-band spectral coverage, both the reflected and the transmitted components have been constrained in a comprehensive picture in which also Compton down-scattering has been taken into account. According to this scenario, the strong absorption is due to transmission through the rim of the torus, while the unabsorbed (or mildly absorbed) reflection component, directly viewed, is due to reprocessing from the torus inner surface. This picture agrees with previous findings for Comptonthick sources (e.g., Matt et al. 2000).

The equivalent width of the iron $\mathrm{K} \alpha$ line, coupled to the absorption column density, allows to constrain the inclination angle of NGC 3281 with respect to the line-ofsight to be higher than $60^{\circ}$ (see Fig. 3 in Ghisellini et al. 1994), assuming typical values for the half-opening angle of the torus and its location in the equatorial plane of the galaxy. A similar value for the inclination angle was suggested by Storchi-Bergmann (1992) from optical observations of this galaxy, in particular from the conical morphology of the high-excitation gas revealed by [O III] $\lambda 5007$ emission. It is worth noting that the conical morphology, even though not peculiar of Comptonthick objects, is expected if large amounts of gas hide the nuclear emission, and was seen in many Sey 2 galaxies (Wilson \& Tsvetanov 1994). Interesting enough, the [O III] flux $\left(\approx 10^{-12} \mathrm{erg} \mathrm{cm}^{-2} \mathrm{~s}^{-1}\right.$, Storchi-Bergmann et al. 1992 ) is about one-third of the $2-10 \mathrm{keV}$ flux derived by BeppoSAX. The $F_{[\mathrm{O} \text { III }]} / F_{2-10 \mathrm{keV}}$ ratio is extremely high if compared to that obtained by Mulchaey et al. (1994) for a sample of Seyfert 2 galaxies, whose average observed ratio is $\approx 0.02$. This provides further support for the hypothesis of obscuration of most of the direct $\mathrm{X}$-rays from NGC 3281 below $10 \mathrm{keV}$.

The derived $N_{\mathrm{H}} / A_{\mathrm{V}}$ ratio is about 50 times higher than the Galactic value, and a factor of $\approx 2$ higher than that derived by Simpson (1998) from the analysis of $A S C A$ data. The high $N_{\mathrm{H}} / A_{\mathrm{V}}$ ratio was originally explained by Simpson (1998) assuming a dense cloud which obscures the entire $\mathrm{X}$-ray source but only partially the more extended infrared emission. An alternative explanation has been recently provided by broad-band studies conducted on AGNs. There is, in fact, growing evidence (e.g., Severgnini et al. 2001; Maiolino et al. 2001a; Risaliti et al. 2001; Vignali 2001) that such high values of $N_{\mathrm{H}} / A_{\mathrm{V}}$ are not exceptions, but seem to be a common property of many AGNs at low and high redshift along three orders of magnitude in $N_{\mathrm{H}}$ (from $10^{21}$ up to $10^{24} \mathrm{~cm}^{-2}$, Guainazzi et al. 2001). Maiolino and collaborators (2001b) have tentatively explained this result assuming larger grain sizes and, as a consequence, a flatter extinction curve. On the contrary, this anti-correlation between $\mathrm{X}$-ray absorption and optical reddening seems not to ubiquitously apply to Compton-thick sources (Guainazzi et al. 2001). This result 
has also cosmological implications. Indeed, if a significant fraction of the $\mathrm{X}$-ray background is not ascribed only to $\mathrm{X}$-ray unabsorbed, broad-line quasars (e.g., Schmidt et al. 1998; Lehmann et al. 2001), but actually also to broadline, dust-free X-ray obscured quasars (e.g., Comastri et al. 2001; Fiore et al. 2001), then the contribution to the IR background of the sources making the hard XRB would be smaller than previously stated (e.g., Fabian \& Iwasawa 1999).

Even though more studies are required to address the above issues, it is however interesting to note that only with a broad-band instrument as the PDS onboard $B e p p o S A X$ it is possible to reveal the nuclear $\mathrm{X}$-ray component in heavily absorbed objects, and to constrain the column density in a accurate way. It should be noted, however, that all three models give a fairly good representation of the $3-100 \mathrm{keV}$ spectrum.

\section{Conclusions}

BeppoSAX observation of the Seyfert 2 galaxy NGC 3281 has allowed to reveal, for the first time, its Comptonthick nature. The $E<10 \mathrm{keV}$ spectrum is reflectiondominated, with a relatively strong $(E W \approx 0.5-1.2 \mathrm{keV})$ iron $\mathrm{K} \alpha$ emission line. The PDS band reveals the presence of the heavily absorbed nuclear continuum, the absorption column density being of the order of 1.5$2 \times 10^{24} \mathrm{~cm}^{-2}$. This finding also justifies the extremely high $F_{[\mathrm{O} \text { III }]} / F_{2-10 \mathrm{keV}}$ value observed in NGC 3281 when compared to the average value for Seyfert 2 galaxies. The $N_{\mathrm{H}} / A_{\mathrm{V}}$ ratio is extremely high (about 50 times the Galactic value), thus adding NGC 3281 to the list of AGNs characterized by dust properties different from the Galactic ones.

The present BeppoSAX results fit well into the unified models scenarios for the Seyfert galaxies and highlight the potentialities of the broad-band X-ray spectroscopy with BeppoSAX to study the buried, heavily absorbed Seyferts, as well as to discover new Compton-thick galaxies.

Acknowledgements. This research has made use of the NASA/IPAC Extragalactic Database (NED) which is operated by the Jet Propulsion Laboratory, California Institute of Technology, under contract with the National Aeronautics and Space Administration. CV wishes to thank G. Matt who allows to use his spectral code for transmission and Compton scattering, G. Brunetti for useful discussions, and the referee for helpful comments. The authors gratefully acknowledge support from the Italian Space Agency, under the contracts ASI 00/IR/103/AS and ASI-ARS-99-75, and from the Ministry for University and Research (MURST) under grant Cofin-00-0236. CV also acknowledges financial support of NASA LTSA grant NAG5-8107.

\section{References}

Anders, E., \& Grevesse, N. 1989, Geochim. Cosmochim. Acta, 53, 197

Antonucci, R. R. J. 1993, ARA\&A, 31, 473
Antonucci, R. R. J., \& Miller, J. S. 1985, ApJ, 297, 621

Arnaud, K. A. 1996, in Astronomical Data Analysis Software and Systems V, ed. G. Jacoby, \& J. Barnes, ASP Conf. Ser., 101, 17

Avni, Y. 1976, ApJ, 210, 642

Bianchi, S., Matt, G., \& Iwasawa, K. 2001, MNRAS, 322, 669

Boella, G., Butler, R. C., Perola, G. C., et al. 1997a, A\&AS, 122,299

Boella, G., Chiappetti, L., Conti, G., et al. 1997b, A\&AS, 122, 327

Bohlin, R. C., Savage, B. D., \& Drake, J. F. 1978, ApJ, 224, 132

Brusa, M., Comastri, A., \& Vignali, C. 2001, in Galaxy Clusters and the High Redshift Universe Observed in X-rays, ed. D. Neumann, F. Durret, \& J. Tran Thanh Van, in press [astro-ph/0106014]

Cagnoni, I, Della Ceca, R., \& Maccacaro, T. 1998, ApJ, 493, 5

Cappi, M., Bassani, L., Comastri, A., et al. 1999, A\&A, 344, 857

Collinge, M. J., \& Brandt, W. N. 2000, MNRAS, 317, L35

Comastri, A., Fiore, F., Vignali, C., et al. 2001, MNRAS, 327, 781

Dickey, J. M., \& Lockman, F. J. 1990, ARA\&A, 28, 215

Done, C., Madejski, G. M., \& Smith, D. A. 1996, ApJ, 463, L63

Fabbiano, G., Kim, D.-W., \& Trinchieri, G. 1992, ApJS, 80, 531

Fabian, A. C., \& Iwasawa, K. 1999, MNRAS, 303, L34

Ferguson, H. C., \& Sandage, A. 1990, AJ, 100, 1

Fiore, F., Guainazzi, M., \& Grandi, P. 1999, Cookbook for BeppoSAX NFI spectral analysis

Fiore, F., et al. 2000, in X-ray Astronomy '999: Stellar Endpoints, AGN, and the Diffuse Background, ed. G. Malaguti, G. G. C. Palumbo, \& N. White, in press [astro-ph/0007118]

Fiore, F., Comastri, A., La Franca, F., et al. 2001, in Proceedings of the ESO/ECF/STSCI Workshop on Deep Fields, ed. S. Cristiani, in press [astro-ph/0102041]

Franceschini, A., Bassani, L., Cappi, M., et al. 2000, A\&A, 353, 910

Frontera, F., Costa, E., Piro, L., et al. 1997, A\&AS, 122, 357

Ghisellini, G., Haardt, F., \& Matt, G. 1994, MNRAS, 267, 743

Goodrich, R. W., Veilleux, S., \& Hill, G. J. 1994, ApJ, 422, 521

Guainazzi, M., \& Matteuzzi, A. 1997, SDC Technical Report

Guainazzi, M., Matt, G., Antonelli, L. A., et al. 1999, MNRAS, 310,10

Guainazzi, M., Matt, G., Brandt, W. N., et al. 2000, A\&A, 356,474

Guainazzi, M., Fiore, F., Matt, G., \& Perola, G. C. 2001, MNRAS, 327, 323

Guainazzi, M., et al., in preparation

Iwasawa, K. 1995, Ph.D. Thesis

Iwasawa, K., Koyama, K., Awaki, H., et al. 1993, ApJ, 409, 155

Iwasawa, K., Matt., G., Fabian, A. C., et al. 2001, MNRAS, 326,119

Lehmann, I., Hasinger, G., Schmidt, M., et al. 2001, A\&A, 371, 833

Madejski, G., Zycki, P., Done, C., et al. 2000, 535, L87

Magdziarz, P., \& Zdziarski, A. A. 1995, MNRAS, 273, 837

Maiolino, R., Salvati, M., Bassani, L., et al. 1998, A\&A, 338, 781 
Maiolino, R., Marconi, A., Salvati, M., et al. 2001a, A\&A, 365, 28

Maiolino, R., Marconi, A., \& Oliva, E. 2001b, A\&A, 365, 37

Malaguti, G., Palumbo, G. G. C., Cappi, M., et al. 1998, A\&A, 331,519

Manzo, G., Giarrusso, S., Santangelo, A., et al. 1997, A\&AS, 122,341

Matt, G., Fiore, F., Perola, G. C., et al. 1996a, MNRAS, 281, L69

Matt, G., Brandt, W. N., \& Fabian, A. C. 1996b, MNRAS, 280,823

Matt, G., Guainazzi, M., Frontera, F., et al. 1997, A\&A, 325, L13

Matt, G., Guainazzi, M., Maiolino, R., et al. 1999a, A\&A, 341, L39

Matt, G., Pompilio, F., \& La Franca, F. 1999b, New Astr., 4, 191

Matt, G., Fabian, A. C., Guainazzi, M., et al. 2000, MNRAS, 318,173

Matt, G. 2001, in Issues in Unification of AGNs, ed. R. Maiolino, A. Marconi, \& N. Nagar, ASP Conf. Ser., in press [astro-ph/0107584]

Miller, J. S., \& Goodrich, R. W. 1990, ApJ, 355, 456

Mulchaey, J. S., Koratkar, A., Ward, M. J., et al. 1994, ApJ, 436,586

Nandra, K., \& Pounds, K. A. 1994, MNRAS, 268, 405

Parmar, A. N., Martin, D. D. E., Bavdaz, M., et al. 1997, A\&AS, 122, 309
Piro, L., de Rosa, A., Dadina, M., et al. 2000, Advances in Space Research, 25, 453

Risaliti, G., Maiolino, R., \& Salvati, M. 1999, ApJ, 522, 157

Risaliti, G., Gilli, R., Maiolino, R., \& Salvati, M. 2000, A\&A, 357,13

Risaliti, G., Marconi, A., Maiolino, R., Salvati, M., \& Severgnini, P. 2001, A\&A, 371, 37

Salvati, M., Bassani, L., Della Ceca, R., et al. 1997, A\&A, 323, L1

Schmidt, M., Hasinger, G., Gunn, J., et al. 1998, A\&A, 329, 495

Severgnini, P., Risaliti, G., Marconi, A., Maiolino, R., \& Salvati, M. 2001, A\&A, 368, 44

Simpson, C. 1998, ApJ, 509, 653

Storchi-Bergmann, T., Wilson, A. S., \& Baldwin, J. A. 1992, ApJ, 396, 45

Tran, H. D. 1995, ApJ, 440, 597

Turner, T. J., George, I. M., Nandra, K., \& Mushotzky, R. F. 1997a, ApJS, 113, 23

Turner, T. J., George, I. M., Nandra, K., \& Mushotzky, R. F. 1997b, ApJ, 488, 164

Turner, T. J., George, I. M., Nandra, K., \& Mushotzky, R. F. 1998, ApJ, 493, 91

Vignali, C. 2001, Ph.D. Thesis, University of Bologna

Vignali, C., Comastri, A., Fiore, F., \& La Franca, F. 2001, A\&A, 370, 900

Vignati, P., Molendi, S., Matt., G., et al. 1999, A\&A, 349, L57

Wilson, A. S., \& Tsvetanov, Z. I. 1994, AJ, 107, 1227 